\title{
Buffer Sizing for QoS Flows in Wormhole Packet Switching NoCs
}

\author{
Leonel Tedesco \\ Itedesco@inf.pucrs.br \\ Ney Calazans \\ calazans@inf.pucrs.br \\ Fernando Moraes \\ moraes@inf.pucrs.br \\ Pontifícia Universidade Católica do Rio Grande do Sul (FACIN-PUCRS)
Av. Ipiranga, 6681 - 90619-900 - Porto Alegre - BRASIL
}

\begin{abstract}
Networks on chip (NoCs) are communication infrastructures that offer parallelism and scalability. Most NoC designs employ wormhole packet switching, since this switching mode optimizes the use of NoC resources. However, this mode may introduce jitter, possibly producing packet loss, due to the violation of temporal QoS constraints. One technique to deal with jitter is to introduce a decoupling buffer (D-buffer) on the target IP. This buffer receives data from the NoC with jitter, while the target IP consumes data from this buffer at the application rate, without jitter. Two problems must be solved to implement D-buffers: (i) which size must the buffer have? (ii) how much buffer space should be filled before data consumption starts (threshold)? This work proposes a general method to define D-buffer size and threshold, considering the influence of packaging, arbitration, routing and concurrency between flows. Before presenting the method, the paper extends a previous traffic model for stream applications and characterizes jitter sources in wormhole packet switching. The experimental results obtained with the proposed method showed that simple traffic models employing constant frame sizes result in small D-buffers. On the other hand, employing video frames from application traces (i.e. real application data) increases buffer size and threshold, still suppressing jitter. Application traces highlight the threshold parameter importance.
\end{abstract}

\section{Categories and Subject Descriptors}

B.7.1 [Integrated Circuits]: Types and Design Styles - advanced technologies, algorithms implemented in hardware, VLSI (very large scale integration).

\section{General Terms}

Design, Experimentation, Measurement, Performance, Theory, Verification.

\section{Keywords}

Networks on Chip, buffer sizing, traffic modeling, quality of service.

Permission to make digital or hard copies of all or part of this work for personal or classroom use is granted without fee provided that copies are not made or distributed for profit or commercial advantage and that copies bear this notice and the full citation on the first page. To copy otherwise, or republish, to post on servers or to redistribute to lists, requires prior specific permission and/or a fee.

SBCCI'07, September 3-6, 2007, Rio de Janeiro, Brazil.

Copyright 2007 ACM 1-58113-000-0/00/0004...\$5.00.

\section{INTRODUCTION}

Some advantages offered by NoCs [1] are that simultaneous transactions may occur between distinct pairs of cores (parallelism) and that connection of new IPs does not imply redesigning the communication infrastructure and cause no significant performance reduction on the overall system (scalability).

Typical data flows occurring in complex SoCs include control signals exchange, large memory block transfers and multimedia streams. Temporal requirements (deadlines to send/receive data) and the data volume to transmit characterize applications. However, the transmission of a given flow through the NoC may modify the original flow rate, resulting in missed deadlines at the target IP. This flow modification is called the load fluctuation phenomenon. Three processes introduce load fluctuation: data packaging, router processing, and concurrency between flows. Variation in latency caused by load fluctuation is called jitter.

One technique to deal with jitter is to introduce a decoupling buffer (D-buffer) on the target IP. This buffer receives data from the NoC with jitter. The target IP consumes data from this buffer, at the application rate, without jitter. Two problems must be solved to implement D-buffers: (i) which size must the buffer have? (ii) how much buffer space should be filled before data consumption starts (threshold)?

Buffer size and threshold must be correctly dimensioned to avoid: (i) data loss, which happens if the NoC cannot write into the Dbuffer (buffer full); (ii) starvation, which happens if the IP has no data to read from the D-buffer (buffer empty); (iii) latency increase, which happens if data consumption by the target IP starts too late (threshold value oversized).

This paper has two main objectives. The first one is to characterize sources of jitter introduced by data packaging, arbitration/routing and concurrency with other flows. The second one is to propose a method for buffer sizing to restore the temporal characteristics of the flow.

This work is organized as follows. Section 2 presents related works in buffer sizing for NoCs. Section 3 presents traffic modeling used for streaming application, and the jitter sources. Section 4 describes the D-buffer sizing method, the main contribution of this work. Section 5 presents experimental results. Section 6 concludes the paper and suggests directions for future works.

\section{RELATED WORK}

Varatkar and Marculescu [2] demonstrate the self-similar characteristic of MPEG traffic, a typical application on current SoCs. The Authors show that it is possible to define the optimal size for buffers of MPEG decoder modules, in order to avoid buffer overflow. In terms of traffic modeling, they present a method for syn- 
thetic traffic generation. In this method, traces of traffic and their statistical properties are combined on a synthetic trace generation procedure. However, experimental traffic scenarios consider only point-to-point communication, discarding an eventual influence of concurrent flows. In addition, there is no mention concerning the buffer threshold value in this work.

$\mathrm{Hu}$ and Marculescu in [3] present a method for buffer sizing on intermediate nodes of NoCs, using formalisms originated from queuing theory. The main objective is to minimize the average latency of all communications occurring on a NoC, while reducing buffer area occupation. The Authors consider data storing in terms of packets, i.e. store-and-forward switching mode. The store-and-forward mode is not frequently used in NoCs, since it increases latency and area, due to the needed buffer size. In terms of traffic modeling, the Authors employ a Poisson synthetic model to characterize telecommunication networks [4]. The disadvantage of this model is the lower accuracy, compared to tracebased models or even self-similar models.

Chandra et al. [5] present a buffer sizing method considering: (i) data production and consumption rates; (ii) size of packets transmitted in burst. The Authors compare throughput when atomic (at the target IP) and distributed (on intermediate nodes) buffers are used. They do not employ statistical properties to the modeled traffic, and the burst sizes are fixed for each simulation. The effect of network concurrency, and consequently jitter introduction, is not mentioned.

Coenen et al. [6] present an algorithm to size buffers at the target IP, in a NoC using virtual channels and credit-based flow control. The objective of this work is to guarantee a constant consumption rate on the target IPs, without data loss. The Authors consider the periodicity properties of both data production and consumption from buffers. They detect this periodicity using two arrays, storing information about data arrival times on the target IP, and the required data processing rate. Again, this work does not consider concurrency between different flows.

In short, the reviewed buffer sizing works do not consider concurrency between distinct flows. Besides, when abstract NoC models (e.g. System-C descriptions) are employed, jitter induced by packaging and arbitration/routing is not taken into account in the sizing methods. This work proposes a general method to define the size and threshold value for $D$-buffers considering the influence of packaging, arbitration, routing and concurrency between flows.

\section{TRAFFIC MODELING AND JITTER SOURCES}

Traffic modeling is the phase on the NoC design cycle where communication requirements are captured [7]. Multimedia applications, such as audio and video streams, represent the typical workload of present SoCs. Works like [2] and [8] describe traffic modeling algorithms for video streams. Streams are composed by frames, generated at constant intervals, characterizing an ON-OFF traffic model. The size of each ON period may vary, as induced for example by the use of compression algorithms. Still, the rate of the ON period is usually constant.

Figure 1 details a packaging process. Figure 1(a) presents the stream under generation by the source IP, started by two frames with different sizes. Each $4 \mu$ s a new frame starts. Assume frames are composed by 16-bit words, with flit size equal to the word size. In the example, frame 0 contains 10 flits and frame 1 contains
30 flits. During each ON period, the IP generates data at a constant rate. In the Figure, the data generation rate for frame 0 and frame 1 is $160 \mathrm{Mbps}$ (10 16-bit flits transmitted in $1 \mu \mathrm{s}$, for frame 0 and 30 16-bit flits transmitted in $3 \mu \mathrm{s}$, for frame1). Figure 1(b) is a detailed view of frame 0 . A flit is produced every 100 ns. For a 50 $\mathrm{MHz}$ clock, both flit generation and consumption should occur every 5 clock cycles. Figure 1(c) shows frame 0 segmented in two packets, with header information included. These packets are injected into the network and stored at a D-buffer on the target IP.

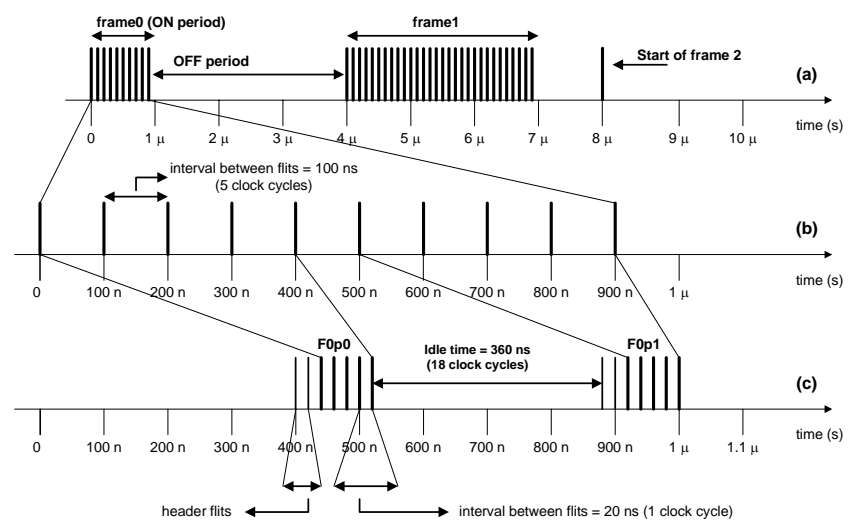

Figure 1 - Data packaging process exemplified using a video stream.

The traffic generation for the ON-OFF traffic model, deals with the following parameters [9]:

- Number of frames, a fixed parameter.

- Frame_start_time, the timestamp of the first payload flit injected into the NoC (440 ns in Figure 1).

- Inter_frames_arrival, a fixed parameter, corresponding to the distance between two consecutive frames.

- Frame_size, which corresponds to the size of the ON period.

- Rate of the $\mathrm{ON}$ period, a fixed parameter. In Figure 1 the rate of the ON period is $160 \mathrm{Mbps}$.

- Number of packets per frame or packet size. If the number of packets per frame is fixed, the packet size varies according to the rate and the ON period size. If the packet size is fixed, the number of packets per frame varies, as illustrated in Figure 1.

\subsection{Jitter Sources}

Three processes introduce jitter in wormhole packet switching NoCs: data packaging, router processing, and concurrency between flows. In contrast, circuit switching does not introduce jitter, and provides latency and throughput guarantees. The reason NoCs adopt wormhole packet switching despite the difficulties to ensure temporal constraints for QoS flows is that wormhole requires no static resource reservation, enabling to optimize NoC resources (e.g. share the same physical channel between two distinct flows).

Due to the higher transmission rate of NoC channels, compared to individual IP core rates, it is necessary to encapsulate application data into packets, in a process called packaging. Incoming flits are stored in a FIFO buffer at the network interface (NI). When the number of stored flits is equal to the packet size, these flits enter the NoC in burst. Header flits occupy the beginning of the packet, containing for example the target router address and the payload size. The idle time between each packet must be respected, to keep the original application data rate. Data packaging 
optimizes channel bandwidth use, due to burst transmission. As illustrated in Figure 1, the addition of the two header flits in the beginning of the packet reduces the idle time between packets from 20 to 18 clock cycles, introducing jitter before injecting packets into the NoC. The effect of header insertion in the jitter depends on the packet size.

This paper assumes the use of distributed routing schemes. In these schemes, a packet injected into the network traverses different routers to reach the target IP. At each intermediate router, a process to select which input port must be served first (arbitration) and the selection of the output port (routing) is executed (only for the header flits). Considering that this process takes some clock cycles to execute, some flits are blocked in the meantime, changing the original packet rate, and consequently increasing jitter. Router designs include input or output buffers to reduce congestion during arbitration and routing, not to reduce jitter.

The third process that introduces jitter is the concurrency between different flows sharing the same NoC resources. When two or more flows are assigned to use the same router output port, one flow transmits first while the others must wait the end of this transmission. This also modifies the original rate of blocked packets, introducing jitter. A technique used to minimize this problem is the adoption of virtual channels, a time division multiplexing method. Virtual channels transmit flits from different flows in an interleaved manner. This technique improves NoC performance, reducing the total latency and the jitter.

Jitter introduces several problems for applications: (i) received data may not respect temporal requirements, such as the interval between bursts; (ii) packet latency increases; (iii) the target IP may lose data. Event though the available bandwidth in NoC channels is usually high (around Gbps [10]), it is necessary to recover the original rate produced by the source IP respecting temporal deadlines. This can be achieved using decoupling buffers, D-buffers, attached to the NI of the target IP.

\section{BUFFER SIZING}

This Section proposes a method to compute the size and the threshold values for the D-buffers. The D-buffers are part of the target IP network interface, as illustrated in Figure 2. The correct definition of the buffer size avoids data loss, and the correct definition of the threshold value avoids starvation. Data loss happens when the NoC cannot write into the D-buffer (buffer full). Starvation happens when the IP has no data to read from the D-buffer (buffer empty). These two parameters are obtained using data collected after the NoC simulation, using the modeled traffic as workload. The simulation data is collected at the output port of each router (data production in Figure 2).

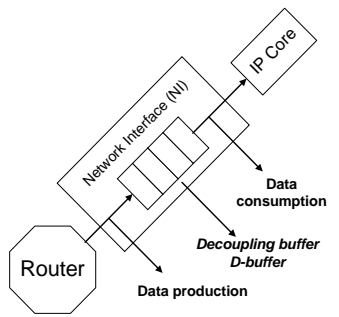

Figure 2 - NI decoupling buffer.

The proposed method starts by filling two arrays. The first array, named recv, collects data generated after simulation at the output port of the target router (data production, in Figure 2). The second array, named cons, is filled according to the rate the IP should receive data. These arrays contains at each index position the value ' 1 ' or ' 0 ' according to the following rules:

- recv[index] = ' 1 ', if a flit is received at the output port at timestamp index, ' $\mathbf{0}$ ' otherwise;

- cons[index] $=$ ' $\mathbf{1}$ ', if a flit must be consumed by the target IP at timestamp index, '0' otherwise.

The size of the recv and cons arrays is equal to the number of clock cycles corresponding to the inter frames arrival (IFA) parameter, which is the time between two frames (Figure 3). Timestamp TR0 corresponds to the timestamp of the first received flit at the router output port. All flits received within the IFA period should belong to the same frame. Flits belonging to a given frame received outside the IFA period are discarded, corresponding to a deadline violation. Figure 6 illustrates the parameters used to fill recv and cons arrays.

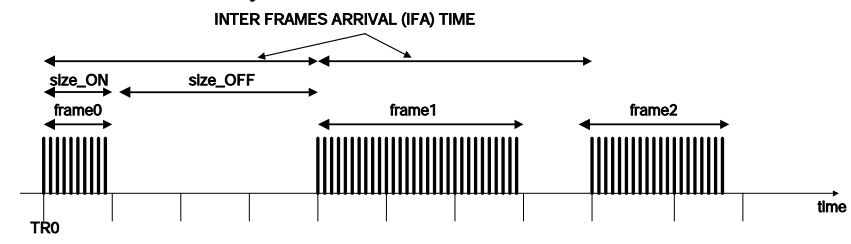

Figure 3 - Parameters for buffer size and threshold definition.

The global counter responsible to count the number of clock cycles, globaltmp, starts counting with the NoC simulation (clock cycle accurate simulation, with the NoC modeled in synthesizable VHDL, and the test bench in SystemC). A new flit arrives at the output port of a given router when the signal outTx is asserted (the assertion of this signal corresponds to the flow control used in most NoCs: handshake or credit-based). For each new flit arrival, the current timestamp (value of the globaltmp counter) is stored in the router timestamp file, named tmpfile.

Figure 4 presents the algorithm to fill the recv array. Tbegin $_{k}$ and Tend $_{k}$, lines 1 and 2, define the interval where flits belonging to the frame $k$ should be received, according to the parameters IFA and TR0. Line 3 initializes the recv array, filling all positions with value ' 0 '. Line 4 starts a loop seeking in the timestamp file, tmpfile, a value within the frame $k$ (line 5 ). The difference between the flit arrival timestamp, value $i$, and the initial frame timestamp, Tbegin $_{k}$, (line 6) defines the recv index. The recv array at the index position receives ' 1 ' (line 7 ). The algorithm finishes when the value read in the tmpfile corresponds to a flit belonging to the next frame (line 9).

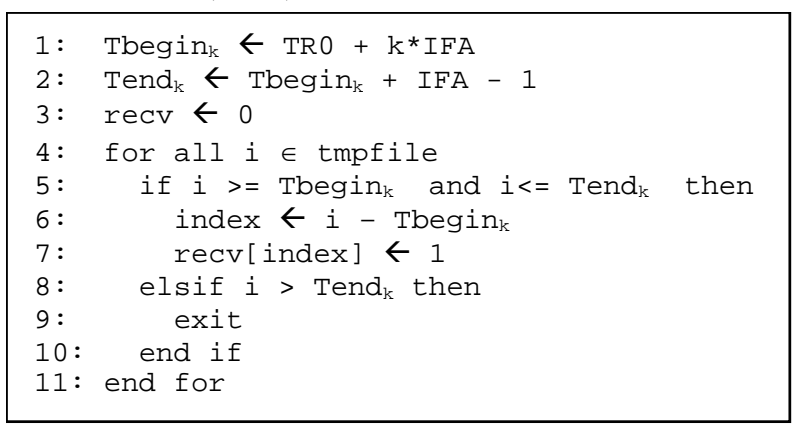

Figure 4 - Algorithm to fill the recv array, for the $k$ th frame.

The consumption array, cons array, considers for each frame $k$ 
the size_ON $N_{k}$ parameter (in clock cycles) and the consumption rate. The consumption rate is normalized to the channel bandwidth. Figure 5 presents the algorithm to fill the cons array. Line 1 initializes the cons array, filling all positions with ' 0 '. Line 2 starts a loop, storing ' 1 ' in the cons array according to the consumption rate.

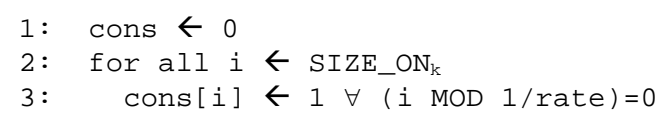

Figure 5 - Algorithm to fill the cons array, for the $k$ th frame.

Figure 6(a) illustrates the execution of the algorithm to fill the recv array. The flits arrival time, stored in the tmpfile, are $\{130$, 133, 134, 138, 141, 145, 146,150, 160\}. Executing the algorithm presented in Figure 8, the recv array is filled according to Figure 6(a). Figure 6(b) illustrates the execution of the algorithm to fill the cons array. The defined size_ON is 16 clock cycles and the consumption rate $50 \%(0.5)$. Each 2 cycles $(1 / 0.5)$ a flit should be consumed from the buffer. In the figure, it is possible to observe that the cons array cells filled with ones are those that are inside the size_ON period. The algorithms are executed for all simulated frames.

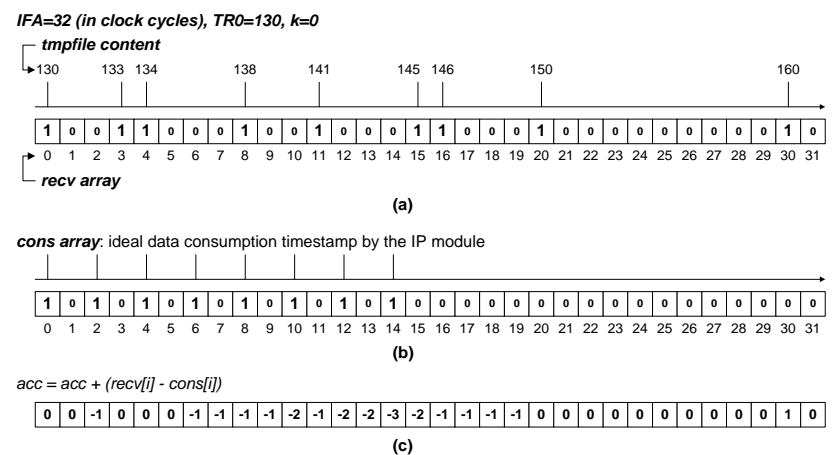

Figure 6 - (a) recv array filling; (b) cons array filling; (c) recv and cons comparison.

The D-buffer size and threshold parameters are computed according to the state of the recv and cons arrays. The following conditions may arrive:

- $\operatorname{recv}[\mathrm{i}]=` 1$ ' $\wedge$ cons[i]= '1': indicates a flit reception and consumption at timeslot $i$.

- $\operatorname{recv}[\mathbf{i}]=' \mathbf{1}$ ' $\wedge$ cons[i]= '0': indicates a flit reception at timeslot $i$, before the moment to consume it. This condition frequently arises in wormhole packet switching NoCs, since flits may arrive in burst after a congestion situation. In this case, a buffer slot is required to store the incoming flit.

- $\operatorname{recv}[\mathbf{i}]=$ ' 0 ' $\wedge$ cons[i]= ' 1 ': the IP must consume a flit at timeslot $i$, but it was not received at this timeslot. This arises when flits are blocked inside the network. In this case, it is necessary to increment the threshold value, to avoid starvation.

The algorithm presented in Figure 7 defines the D-buffer size and threshold values. It computes the instantaneous difference, acc value, between the two vectors (line 3 ) for all timestamps, and the maximal (lines 4-6) and minimal (lines 7-9) values of acc. The Dbuffer size corresponds to the addition of the $a c c$ maximum value with its absolute minimal value (line 12).The absolute minimum value of acc indicates how many flits must be stored in the Dbuffer before start consumption, i.e. the threshold value (line 13).

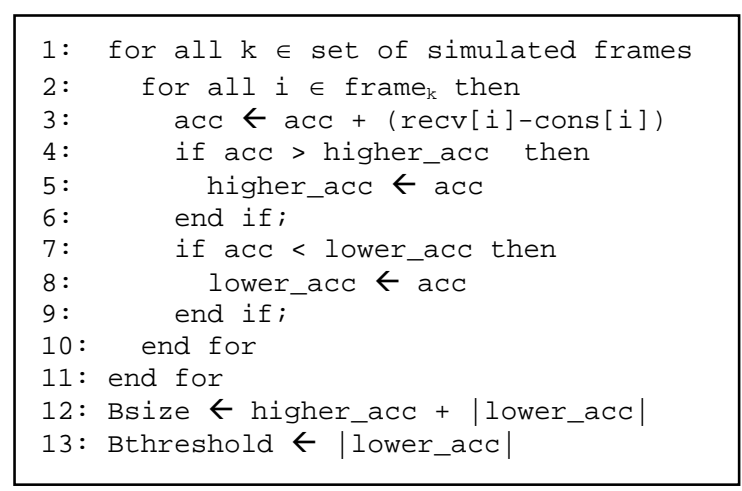

Figure 7 - Algorithm to define the size and threshold values for the D-buffer.

Figure 6(c) presents the acc value when executing the algorithm detailed in Figure 7. The execution of the algorithm results in lower_acc $=-3$ and higher_acc $=1$. Therefore, Bsize is equal to 4 and Bthreshold is equal to 3. The D-buffer sized according to this method enables the IP to consume the flits according to the specified rate, without data loss. The threshold value becomes a design parameter, included in the NI of the target IP. When the number of received flits reaches the threshold value, the IP may safely read the D-buffer.

\section{RESULTS}

The HERMES [11] NoC was used to evaluate the traffic modeling and the buffer sizing technique proposed in this work. The fixed network design parameters are: $8 \times 8$ mesh; credit-based flow control; 16-bit flit size; 8-flit depth internal buffers; deterministic XY routing algorithm; 2 virtual channels associated to each physical channel. The employed NoC frequency is $50 \mathrm{MHz}$, corresponding to a channel rate of $800 \mathrm{Mbps}$.

The experiments employ three synthetic traffic models (Table 1). Control signals are used as noise traffic, being transmitted using a complement spatial distribution by all IPs not transmitting HTTP or HDTV traffic. HTTP traffic is used to disturb the real time streaming traffic, HDTV. The HDTV traffic is characterized with temporal requirements.

Table 1 - Traffic modeling for the conducted experiments (PS: packet size, IR: Injection Rate).

\begin{tabular}{|c|c|c|l|}
\hline Application & Requirement & \multicolumn{2}{|c|}{ Traffic characteristics } \\
\hline $\begin{array}{c}\text { Control Sig- } \\
\text { nals }\end{array}$ & Signaling & PS & 15 flits \\
\cline { 3 - 4 } 100 HTTP & IR & Constant injection rate: 16 Mbps \\
\hline sessions & $\begin{array}{c}\text { Real-time block } \\
\text { transfer }\end{array}$ & PS & $\begin{array}{l}\text { 600 to 1500 flits (fixed sized per } \\
\text { experiment) }\end{array}$ \\
\cline { 3 - 4 } 10 HDTV & IR & $\begin{array}{l}\text { Pareto ON-OFF: 160 Mbps ON } \\
\text { period }\end{array}$ \\
\hline channels & $\begin{array}{c}\text { Real time } \\
\text { streaming }\end{array}$ & PS & $\begin{array}{l}\text { Fixed (1500 flits) and trace- } \\
\text { based (1500 flits on average) }\end{array}$ \\
\cline { 3 - 4 } & IR & $\begin{array}{l}\text { Transmission of 10 simultane- } \\
\text { ous HDTV flows per frame. } \\
\text { Rate during a frame period: } 200 \\
\text { Mbps (25\% load) }\end{array}$ \\
\hline
\end{tabular}

Control signal packets are modeled with small 15-flit packets, which represent a typical size for this type of traffic. These packets are continuously generated in time, characterizing a CBR flow. The injection rate is $16 \mathrm{Mbps}$, corresponding to $2 \%$ of channel bandwidth. HTTP traffic is modeled using Pareto ONOFF injection process, where packets are generated during the ON period at $160 \mathrm{Mbps}$. HDTV traffic is modeled using the described ON-OFF model. The ON period generates 10 packets at 
200 Mbps, corresponding to 10 HDTV channels, consuming 25\% of a NoC channel bandwidth. Each HDTV initiator transmits 20 frames. The simulated scenarios employ fixed-size or tracedbased packets [12].

Figure 8 illustrates a first spatial traffic distribution, with two HDTV initiators (M1-M2), with the HDTV flow M1 disturbed by three HTTP flows. The complement distribution employed by the noise traffic increases the communication volume on the network bisection [9]. HDTV and HTTP flows are placed next to the middle of the network, to maximize the noise traffic influence in these flows.

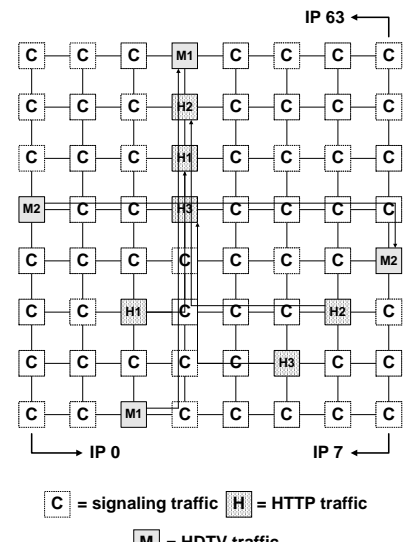

$M=$ HDTV traffic

Figure 8 - Spatial traffic distribution, with HDTV M1 being disturbed by three HTTP flows.

\subsection{Simulation Scenario 1: Frames With Fixed Packet Sizes}

This scenario models HDTV flows with the ON-OFF traffic model, using fixed-size packets, with size varying between 500 and 1500 flits. Table 2 presents the results.

Table 2 - Simulation results for HDTV flow with fixed-size packets.

\begin{tabular}{|c|c|c|c|c|c|}
\hline $\begin{array}{c}\text { HDTV } \\
\text { packet } \\
\text { size }\end{array}$ & $\begin{array}{c}\text { HTTP } \\
\text { sources }\end{array}$ & $\begin{array}{c}\text { HTTP } \\
\text { packet } \\
\text { size }\end{array}$ & $\begin{array}{c}\text { jitter } \\
\text { (HDTV) }\end{array}$ & $\begin{array}{c}\text { threshold } \\
\text { (HDTV) }\end{array}$ & $\begin{array}{c}\text { buffer } \\
\text { size } \\
\text { (HDTV) }\end{array}$ \\
\hline 500 & \multicolumn{2}{|c|}{ No concurrency } & 0 & 0 & 375 \\
\hline 500 & 2 & 250 & 0.23 & 0 & 478 \\
\hline 1000 & 2 & 500 & 0.33 & 0 & 944 \\
\hline 1500 & 3 & 750 & 0.38 & 0 & 1427 \\
\hline
\end{tabular}

The second line of Table 2 presents one HDTV flow without concurrent HTTP flows nor noise traffic. Since there is no concurrency, all flits arrive at the D-buffer with the same latency, resulting in a null jitter. The threshold value (0) means that flits can be consumed as soon as they arrive at the D-buffer. Two reasons explain this fact: (i) as all packets have the same size, the idle time between packets is the same, resulting in enough time to consume the flits; (ii) the consumed bandwidth is inferior to the channel bandwidth, thus NoC channels do not saturate. The HDTV application rate takes $25 \%$ of the channel bandwidth, meaning that one flit must be consumed each 4 clock cycles. Therefore, for each consumed flit, three flits remain stored in the $D$-buffer. Multiplying the packet size, 500 , by 0.75 (percentage of flits not consumed), the result is 375 , the obtained buffer size.

Lines three to five of Table 2 present one HDTV flow competing with two HTTP flows and noise traffic. Flits arrive at the D-buffer with a jitter varying between $23 \%$ and $38 \%$. The previously dis- cussed factors introduce jitter, which is also influenced by the packet size. Even with concurrency between flows, the threshold value remains zero. Again, fixed packet sizes and application rates inferior to the channel rate explain this value. However, the concurrency induces a buffer size bigger than the relationship "packet size"/“stored flits", used when there is no concurrency.

Figure 9 shows the number of filled buffer slots ( $x$ axis) versus the number of clock cycles these slots are filled during a simulation run ( $y$ axis). In the absence of concurrency, the buffer is homogeneously used in time. This behavior changes with concurrent traffic. When a given packet is blocked, it is possibly sent with a smaller idle time w.r.t. the next packet, in this way requiring buffer space to avoid deadline violations. Comparing the D-buffer sizes $\left(6^{\text {th }}\right.$ column, Table 2$)$ of the second and third rows, the difference is 103 , corresponding to the right shifting of the curve. These 103 extra buffer slots are required to absorb the jitter introduced by the concurrency between flows.

This first experiment demonstrated the effect of the concurrency between flows in the jitter and buffer size, even when virtual channels are employed. The curves of Figure 9 were obtained simulating the network with the D-buffer, after sizing it. The number of lost packets was zero, with a constant buffer consumption rate, validating the method to size the buffers.

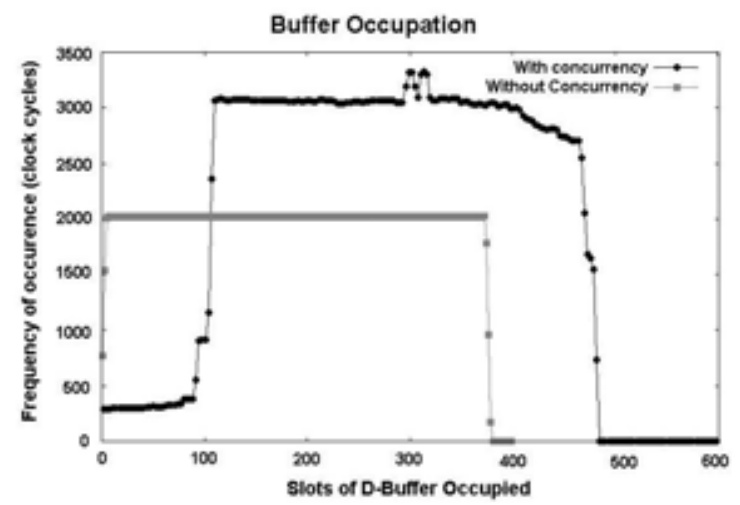

Figure 9 - D-buffer occupation using HDTV with fixed packet sizes, 500-flit packets HDTV flow without (gray) and with (black) concurrency.

\subsection{Simulation Scenario 2: Frames Sizes from Traffic Traces}

The second experiment models the HDTV flows with the ONOFF traffic model, employing packets with variable size, obtained from traffic traces [12]. Traces have an average packet size equal to 1500 flits, varying from a minimum of 15 flits and a maximum of 9066 flits. Table 3 presents the simulation results.

Table 3 - Simulation results for HDTV flow with packets with variable size.

\begin{tabular}{|c|c|c|c|c|c|}
\hline $\begin{array}{c}\text { HTTP } \\
\text { sources }\end{array}$ & $\begin{array}{c}\text { HTTP } \\
\text { packet } \\
\text { size }\end{array}$ & $\begin{array}{c}\text { jitter } \\
\text { (HDTV) }\end{array}$ & $\begin{array}{c}\text { threshold } \\
\text { (HDTV) }\end{array}$ & $\begin{array}{c}\text { higher_acc } \\
\text { (HDTV) }\end{array}$ & $\begin{array}{c}\text { buffer } \\
\text { size } \\
\text { (HDTV) }\end{array}$ \\
\hline \multicolumn{2}{|c|}{ No concurrency } & 0 & 4043 & 640 & 4683 \\
\hline 2 & 600 & 0.25 & 4045 & 640 & 4685 \\
\hline 2 & 2000 & 0.43 & 3436 & 1251 & 4687 \\
\hline 3 & 750 & 0.4 & 4053 & 651 & 4692 \\
\hline 3 & 1000 & 0.45 & 4036 & 651 & 4687 \\
\hline
\end{tabular}

The second line of Table 3 presents an experiment with one HDTV flow without concurrent flows. The jitter between packets is zero, as in the previous scenario. Note the threshold parameter, 
which is now 4043. The threshold is greater than zero, since real application data traces contain different packet sizes. Figure 10 shows packets arriving at the D-buffer, being consumed by the application, considering threshold value equal to zero. The interval between packets is proportional to the packet size. As packet 2 is longer than packet 1 , the interval between these two packets is longer than the required time to consume packet 1 , causing an interval gap between packets. This gap corresponds to a starvation condition, since the IP has no data to consume. The threshold value solves this problem, since it defines the amount of flits to be stored in the D-buffer before data consumption starts. This value is high because a significant variation in packet length is present in the trace files.

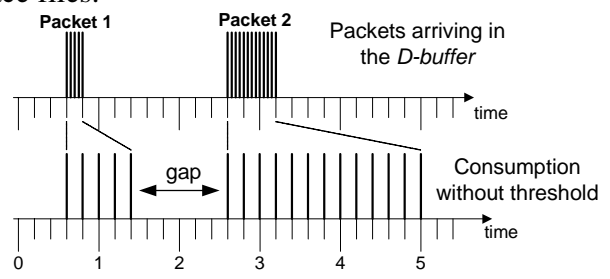

Figure 10 - Data consumption without the threshold parameter, causing starvation.

The column higher_acc corresponds to the amount of flits that must be stored to avoid buffer overflow (Figure 7), as in the first scenario. The buffer size value corresponds to the addition of the threshold and higher_acc parameters. The third and fourth lines of Table 3 show the trace-based HDTV with the concurrency of two HTTP flows and traffic noise. These experiments display an import jitter, varying from $25 \%$ to $43 \%$. It is important to observe that the higher_acc value increases with the packet size of the disturbing flows (600 flits in the third line and 2000 in the fourth). The fifth and sixth lines show an experiment with 4 concurrent flows, 3 HTTP and one HDTV. These experiments show similar results, with an important jitter (40\% to $45 \%$ ).

Figure 11 presents the buffer use for a flow having variable packet sizes. As shown in Figure 9, flows with fixed packet sizes use the buffer in a uniform way. Figure 11 shows a distinct behavior, since the buffer occupation is in average $70 \%$ during the application simulation. This is induced by larger packets, which must be buffered in order to allow the consumption at the application rate.

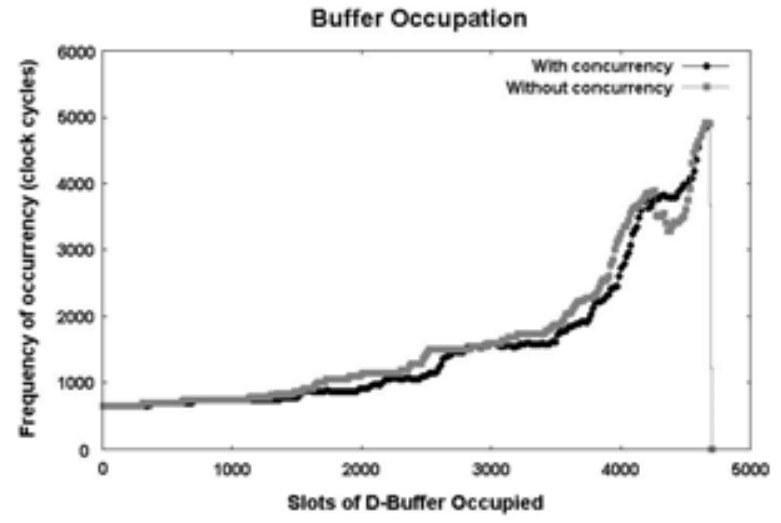

Figure 11 - D-buffer using HDTV with variable packet sizes, HDTV flow without (gray) and with (black) concurrency of 750-flit HTTP packets.

\section{CONCLUSIONS AND FUTURE WORK}

This work presented a method to define the size of decoupling buffers ( $D$-Buffers), attached to the NoC output ports, to minimize the effect of jitter occurrence on a given traffic. Two parameters characterize decoupling buffers: size and threshold. The use of decoupling buffers is necessary for applications that process data periodically in time, in contrast with aperiodic data reception from NoC. The obtained results show that modeling data traffic with fixed frames sizes leads to underestimating buffer occupation. The use of trace based traffic models allows the estimation of buffers considering the inherent variability of streaming data, which makes necessary a higher level of threshold, compared to estimations based on synthetic traffic models.

Another parameter evaluated during simulation was buffer occupation during simulation. Results indicated that during most of the simulation run, the buffer occupation is in average $70 \%$. Results indicated that the buffer occupation was near $100 \%$ during most of simulation run, caused by larger HDTV packets. Thus, depending on application requirements (e.g. tolerance on data losses), it is possible to use only part of the obtained size without significant effect on application behavior.

Future work includes developing a technique to estimate the recv array contents, based on statistical properties of the incoming traffic. This can be useful, considering that the method presented in this work estimates buffer dimensioning based in all data that arrives on the target IP, being time-consuming.

\section{ACKNOWLEDGMENTS}

This research was supported partially by CNPq (Brazilian Research Agency), project 300774/2006-0.

\section{REFERENCES}

[1] Benini, L.; De Micheli,G. "Networks on chips: a new SoC paradigm". IEEE Comp., v.35(1), 2002, pp. 70-78.

[2] Varatkar, G.; Marculescu, R. "On-Chip Traffic Modeling and Synthesis for MPEG-2 Video Applications”. IEEE Transactions on VLSI Systems, 12(1), 2004, pp. 108-119.

[3] Hu, J. et al. "System-Level Buffer Allocation for ApplicationSpecific Networks-on-Chip Router Design”. IEEE Transactions on Computer-Aided Design of Integrated Circuits and Systems, 25(12), 2006, pp. 2919-2933.

[4] Frost, V.; Melamed, B. "Traffic Modeling for Telecommunications Networks”. IEEE Communications Magazine, 32(3), 1994, pp. 7081.

[5] Chandra, V. et al. "An interconnect channel design methodology for high performance integrated circuits”. In: DATE`04, pp. 1138-1143.

[6] Coenen, M. et al. "A Buffer-Sizing Algorithm for Networks on Chip using TDMA and Credit-Based End-to-End Flow Control.”. In: CODES+ISSS’06, pp. 130-135.

[7] Tedesco, L. et al. "Application driven traffic modeling for NoCs". In: SBCCI’06, pp. 62-67.

[8] Hu, J.; Marculescu, R. "Dyad - Smart routing for networks on chip". In: DAC’04, pp. 260-263.

[9] Tedesco, L. et al. "Traffic Generation and Performance Evaluation for Mesh-based NoCs ”. In: SBCCI’05, pp. 184-189.

[10] Guerrier, P.; Greiner, A. “A Generic Architecture for On-Chip Packet-Switched Interconnections”. In: DATE’00, pp. 250-256.

[11] Moraes, F. et al. "Hermes: an Infrastructure for Low Area Overhead Packet-switching Networks on Chip”. Integration, the VLSI Journal, 38(1), 2004, pp. 69-93.

[12] Volisz, A. et al. "MPEG-4 and H.263 Video Traces for Network Performance Evaluation”. http://www-tkn.ee.tu-berlin.de/research/ trace/trace.html. Captured in 2007. 